\title{
UN HYBRIDE POUR LA SALMONICULTURE EN EAUX FROIDES : L'OMBLE-SAUMON
}

\author{
par P. CHIMITS \\ Ingénieur principal des Eaux et Forêts, à Pau.
}

En matière agricole, les génétistes cherchent par l'hybridation un produit ayant, en principe et à la limite, les qualités des deux parents sans en avoir les défauts. Le mulet, par exemple, a la force du cheval tout en ayant la rusticité de l'âne et est adapté aux conditions de la montagne.

En matière d'élevage piscicole, Russes et Américains effectuent, depuis une dizaine d'années, des recherches d'hybridation sur des espèces et dans des buts d'ailleurs différents. Les Russes ont récemment annoncé avoir réussi un hybride fécond de deux espèces de la famille des Esturgeons : le grand Esturgeon et le Sterlet, hybride chez lequel ils espèrent trouver l'abondance de reproduction du Sterlet avec la croissance et les dimensions du grand Esturgeon. Les Américains se sont livrés, ces dernières années, à des essais de croisement entre les diverses espèces de salmonidés : Truite arc-en-ciel, Saumon de fontaine, Cristivomer namaycush en y ajoutant également la Truite commune introduite d'Europe. Leur but était d'obtenir un produit fécond ayant de bonnes qualités pour l'élevage et plus exactement une bonne croissance et une bonne résistance aux maladies. Ci-dessous un tableau des résultats obtenus par les spécialistes en cette matière de croisement (d'après Buss et Wright) :

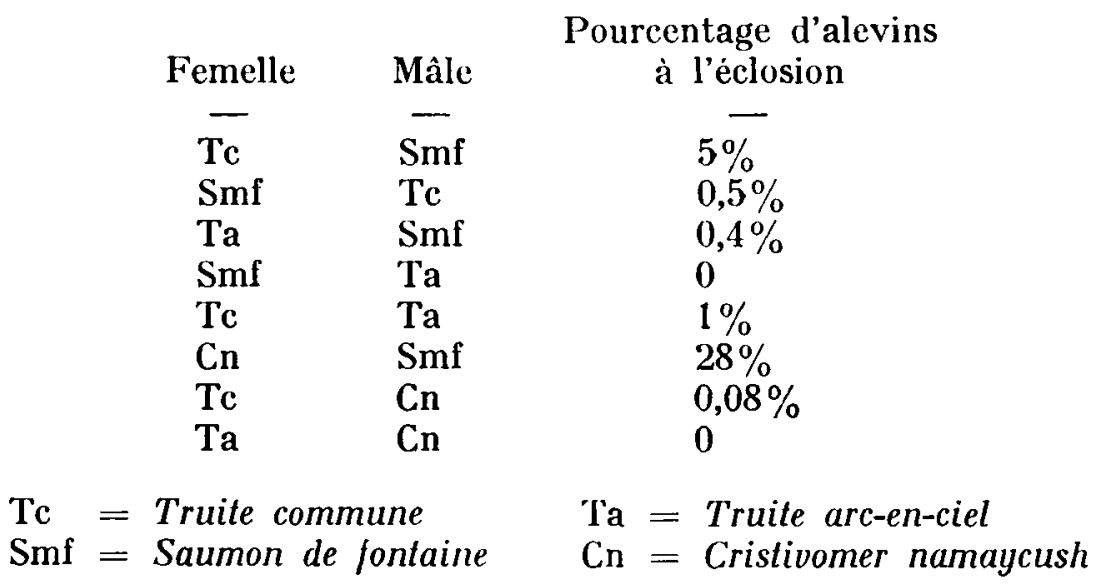


Il résulte de ce tableau que seuls les croisements Cristivomer avec Saumon de fontaine donnent un pourcentage à l'éclosion relativement satisfaisant de $28 \%$ au lieu de 70 à $80 \%$ normalement obtenu par fécondation artificielle entre géniteurs de la même espèce.

De fait, après 10 ans d'essais (commencés vers 1950), les Américains et Canadiens concentrent leurs recherches sur ce seul hybride Saumon de fontaine $\times$ Cristivomer (soit 2 salvelinus) qui présente les qualités ci-dessous :

$1^{\circ}$ Il est fécond et donne en $2^{\mathrm{e}}$ génération, à la fécondation entre hybrides, des résultats très honorables de l'ordre de grandeur de $70 \%$.

$2^{\circ}$ C'est un beau poisson ayant une meilleure croissance en élevage industriel que le Saumon de fontaine, lequel est à peu près aussi utilisé en pisciculture américaine que l'arc-en-ciel, alors que les Européens élèvent surtout l'arc-en-ciel.

$3^{\circ}$ Il semble avoir la résistance du Cristivomer aux maladies et on sait que les maladies font de plus en plus de ravages dans les élevages industriels tant américains qu'européens.

A noter que le seul salmonide non américain, la Truite commune originaire d'Europe, utilisé dans ces croisements, n'a donné que des résultats pratiquement peu intéressants (5\% de réussite à l'hybridation).

A noter également que, aussi bien Russes qu'Américains et Canadiens, se sont uniquement attachés à rechercher des hybrides féconds.

\section{Essais d'hybridation a la Pisciculture Domaniale de Cauterets.}

Les inconvénients de la salmoniculture en eau trop froide.

C'est au cours de la campagne 1956-1957 qu'au courant des essais américains, nous avons commencé les premiers essais d'hybridation avec le matériel de géniteurs de salmonidés dont nous disposions à la pisciculture de Cauterets.

Notre attention avait été attirée par les difficultés rencontrées par des pisciculteurs professionnels utilisant des eaux très froides, notamment dans les piscicultures de montagne, aussi bien des Alpes que des Pyrénées. Alors que ces pisciculteurs sont fort bien placés, grâce au tourisme, pour la vente de leurs produits à la belle saison, leurs élevages se heurtent :

10 A la faible croissance de leurs élèves en eau très froide, la Truite arc-en-ciel ayant besoin de 24 à 36 mois pour atteindre la taille-portion de 150 grammes, au lieu de 12 à 24 mois en élevage de plaine.

$2^{\circ}$ Aux maladies plus fréquentes en eau très froide et ce d'autant plus que le cycle vital de la Truite est deux fois plus long qu'en plaine.

On conseille bien à ces pisciculteurs d'élever le Saumon de fontaine, croissant en eau froide un peu plus vite que la Truite arc-en-ciel, mais il est en revanche plus sensible aux maladies que cette dernière, notamment au goître et à la pourriture des nageoires. 
$3^{0}$ Aux inconvénients de la maturation sexuelle hâtive. L'inconvénient qui nous a semblé le plus grave dans ces piscicultures à eau froide et que nous n'avons jamais vu signaler par les auteurs est que, à la fin de sa $2^{\mathrm{e}}$ année, parfois même dès sa $1^{\text {re }}$ année, le Saumon de fontaine mâle est sexuellement mûr : d'où chaque année, pendant 3 mois, retard de croissance entraîné par l'élaboration des produits génitaux, amaigrissement et mortalité due aux batailles entre mâles et aux maladies extérieures venant sur les blessures.

Cet inconvénient de la maturation sexuelle hâtive ne se rencontre chez la Truite arc-en-ciel qu'à partir de la $2^{\mathrm{e}}$ et de la $3^{\mathrm{e}}$ année et est donc moins sensible dans les piscicultures à eau tempérée où la tailleportion est généralement atteinte à moins de 24 mois.

\section{L'hybridation, moyen pratique de castration biologique.}

C'est cette idée de la suppression de la maturité sexuelle avant la taille-portion, gros inconvénient des piscicultures d'eau froide, qui nous a guidé dans nos essais d'hybridation : un hybride infécond, du seul fait de sa stérilité, doit à priori avoir une meilleure croissance et une meilleure résistance aux maladies pendant les 3 mois d'activité sexuelle normale en hiver. En d'autres termes, en la matière, fertilité s'oppose dans une certaine mesure à croissance et à résistance aux maladies, et, de même qu'en matière générale d'élevage pour une meilleure production de viande, on castre les animaux, soit par moyen physique (bovins, porcins), soit par moyen chimique (volailles par injection d'hormones sexuelles), le même résultat peut être obtenu chez les poissons par castration par un moyen biologique, l'hybridation. Si, dans le cas du mulet, l'hybridation entre âne et cheval est obtenue par la même méthode, il faut bien souligner qu'elle n'est pas recherchée à priori, l'engraissement chez le mulet ne présentant pas d'intérêt. Au contraire, dans notre essai de salmoniculture, nous avons recherché la stérilité, c'est-à-dire la castration biologique comme technique d'un meilleur grossissement.

Ce point capital, surtout pour l'élevage à croissance lente en eau froide, ne semble pas avoir été aperçu par les Américains et les Canadiens qui ont recherché croissance et résistance aux maladies uniquement dans les qualités intrinsèques de l'hybride dont, en même temps, ils recherchaient la fertilité.

\section{Les conditions physiques des eaux de Caulerets et le cheptel de géniteurs.}

La pisciculture domaniale de Cauterets est orientée, à partir d'un cheptel important de géniteurs de 5 espèces de salmonidés d'eau froide, vers la production d'œufs embryonnés et d'alevins destinés par leur préadaptation au repeuplement des eaux froides de montagne.

L'eau y est très pure (degré hydrotimétrique 1) et acide $(p H 6,2)$ car elle provient de sols exclusivement granitiques. Elle y est également très froide $\left(2\right.$ à $3^{\circ}$ pendant l'hiver, 4 à $5^{\circ}$ pendant 4 autres mois, atteignant au maximum 11 à $12^{\circ}$ pendant 2 ou 3 mois l'été). 
Dans ces conditions, la croissance y est lente et la taille portion de 150 grammes n'y est atteinte qu'à plus de deux ans chez la Truite arcen-ciel et le Saumon de fontaine et à plus de 3 ans chez la fario. Une pisciculture industrielle destinée à faire des Truites portion serait donc peu rentable à Cauterets. La température est même trop froide pour une croissance normale, non seulement de Truite arc-en-ciel, mais même du Saumon de fontaine, et ne correspond à l'optimum que pour l'Omblechevalier qui se trouve bien dans les eaux à 6 ou $8^{\circ}$ mais se prête mal à la captivité et à l'élevage.

\section{Les croisements obtenus à Caurerets. L'Omble-saumon.}

Nous ne relaterons pas ici nos divers essais de croisement entre les deux espèces européennes, Truite commune et Omble-chevalier, et les 3 espèces américaines Truite arc-en-ciel, Saumon de fontaine et Cristivomer namaycush, stabulées à Cauterets, et nous arrivons de suite aux 2 seuls essais réussis, tous deux à base de Saumon de fontaine femelle considéré comme l'espèce d'élevage de base en eau froide et dont nous avons voulu améliorer les qualités par le croisement mâle Cristivomer et mâle Omble-chevalier, espèces d'eau très froide.

Le croisement Saumon de fontaine femelle $\times$ Cristivomer mâle (le brookinaw des Américains) commencé en 1960, a donné un résultat à l'embryonnement de $50 \%$, ce qui est légèrement supérieur aux résultats américains mais a l'inconvénient de donner un hybride fécond, ce qui ne nous intéresse pas. Et d'ailleurs, ce croisement et son inverse, le " moulac " des Canadiens, est suffisamment étudié par les Américains et les Canadiens.

Nous retiendrons ici le croisement Saumon de fontaine femelle $\times$ Omble-chevalier mâle, c'est-à-dire le croisement d'une espèce américaine avec une espèce européenne, effectué pour la première fois pendant l'hiver 1956-57. A noter que le Saumon de fontaine à 84 chromosomes et l'Omble-chevalier 80 seulement.

G. Alm, en Suède, avait déjà réussi l'hybridation Saumon de fontaine $\times$ Omble-chevalier, avec un pourcentage de réussite égal au nòtre $(50 \%$ ), mais le produit était fécond. Demoll et SteinmanN (cités par Vivier) indiquent également que cet hybride Saumon de fontaine $\times$ Omble-chevalier, est fécond. Celui que nous avons obtenu à Cauterets est stérile, du moins pendant les 5 à 6 premières années.

Nous avons donc à la pisciculture de Cauterets des sujets hybrides, àgés de 6 ans, et dépassant le kilo. Ce sont des poissons magnifiques, ayant l'allure générale du Saumon de fontaine avec un dos vert clair entièrement zébré de vermiculations blanc jaunâtre bien plus prononcées que chez le Saumon de fontaine et bien plus étendues, s'étalant jusqu'au bas des flancs. Au contraire du Saumon de fontaine, l'hybride ne comporte aucun point rouge sur le flanc. Les nageoires ont la dimension de celles du père Omble-chevalier; elles sont longues, gris cendré, avec un liseré blanc sauf aux pectorales, la nageoire dorsale étant entièrement tigrée.

Nous avons renouvelé chaque année l'expérience et sommes en pos- 
session d'une collection complète de ces hybrides. Leur élevage en pisciculture domaniale de Cauterets a permis de faire les observations cidessous sur cet hybride Saumon de fontaine femelle $\times$ Omble-chevalier mâle (Salvelinus fontinalis $\times$ Salvelinus alpinus).

$1^{\circ}$ La croissance en eau très froide (20 l'hiver, $12^{\circ}$ l'été) est égale à celle du Saumon de fontaine pendant la période hors activité sexuelle.

$2^{\circ}$ La résistance aux maladies est meilleure que chez le Saumon de fontaine pur. Nous n'avons en effet noté aucun cas de goître à la thyroïde ni de pourriture des nageoires, maladies fréquentes chez le Saumon de fontaine d'espèce pure. Ayant diagnostiqué, l'été 1962, une épidémie

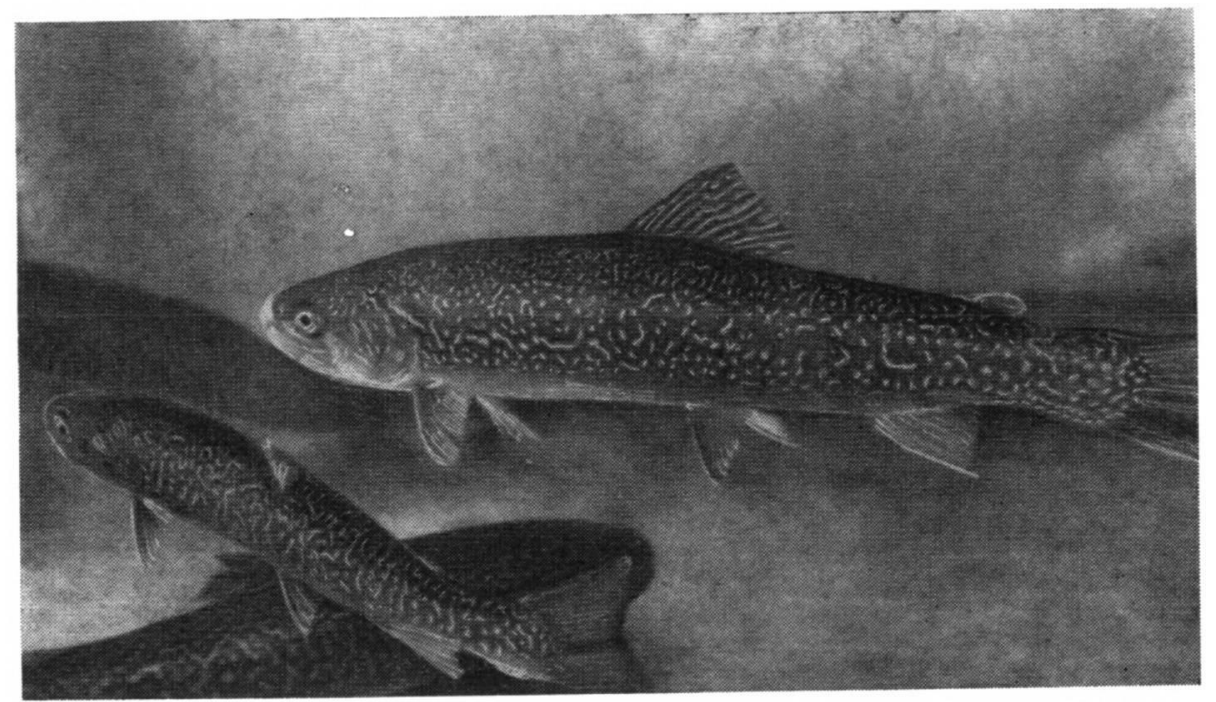

Pisciculture domaniale de Cauterets.

Iybrides Saumon de fontaine femelle $\times$ Omble-chevalier male, agés de 6 ans et pesant $1 \mathrm{~kg}$.

de furonculose que nous avons enrayé par un traitement au chloramphénicol, nous avons pu observer une forte mortalité chez le Saumon de fontaine et une assez faible mortalité chez l'hybride.

$3^{\circ}$ Enfin et surtout, âgés de 6 ans, les hybrides n'ont manifesté aucune activité sexuelle et par conséquent ni retard de croissance ni blessure provenant de batailles entre mâles.

Tels sont les résultats obtenus à Cauterets au seul échelon expérimental. Il reste à faire vérifier ces résultats en pisciculture industrielle d'eau très froide. Ayant opéré, fin octobre 1962, une hybridation sur 30.000 cufs de Saumon de fontaine, soit :

- 15.000 œufs de Saumon de fontaine croisés par Omble-chevalier - réussite à l'embryonnement 7.000 - rendement $47 \%$;

- 15.000 œufs de Saumon de fontaine croisés par Cristivomer réussite à l'embryonnement 7.500 - rendement $50 \%$. 
ces œufs ont été adressés, avec autorisation de l'Administration, à la pisciculture industrielle "Salmoniculture de la Haute Vallée de l'Aude " (Aude) dont les eaux sont très froides et où on élève exclusivement le Saumon de fontaine. Il ne reste plus qu'à attendre 2 ans pour voir si les résultats obtenus en pisciculture industrielle corroborent ceux obtenus en pisciculture expérimentale.

Mais nous avons tenu à indiquer dès à présent les résultats des 6 années d'expériences auxquelles nous ne pouvons plus prendre part, n'étant plus chargé de la gestion de la pisciculture de Cauterets, pour souligner l'intérêt présenté en pisciculture industrielle par un hybride infécond obtenu par croisement de deux espèces géographiquement fort éloignées (une européenne, une américaine) dès lors que l'hybridation peut être obtenue avec une réussite de $50 \%$.

Une telle proportion comparée avec la réussite normale de $80 \%$ en fécondation à l'intérieur d'une même espèce, ne grèvera que très faiblement le prix de revient des poissons-portion, inconvénient compensé bien au-delà par la résistance aux maladies et la meilleure croissance dues précisément à la stérilité du nouvel hybride. Un autre avantage à cette castration par voie biologique est l'absence du choc opératoire ou hormonal consécutif à la castration par voie physique ou chimique.

\section{NOTE DE L'AUTEUR}

M. Jacques Gallon, Directeur de la Salmoniculture de la Haute Vallée de l'Aude, nous communique les premiers résultats au bout de 3 mois de l'élevage industriel des lots d'hybrides Omble-chevalier $\times$ Saumon de fontaine et Cristivomer $\times$ Saumon de fontaine, et de l'espèce pure Saumon de fontaine, que nous lui avons adressés.

L'éclosion est plus longue chez l'hybride Omble $\times$ Saumon $(9$ jours) que chez l'hybride Cristivomer $\times$ Saumon et le Saumon de fontaine $(4$ et 3 jours).

Les pertes $\mathrm{du} 1^{\text {er }}$ âge sont également nettement plus élevées et le début de l'élevage plus difficile et c'est là un inconvénient sérieux.

A partir du $2^{e}$ mois, il y a stabilisation et les alevins Omble $\times$ Saumon sont en bonne santé et s'accroissent normalement. 
BIBLIOGRAPHIE

ALy (G.). - 1954. Artificial hybridization between different species of the salmon family. Reports, Swedish State Institute of Fresh Water Fishery Research $36-13-56$.

Bess Keen and J. C. Wright. -- 1956. Results of species hybridization with the family salmonidae. Progressive lish Culturist 18 (1).

Sowards (Charles L.). -- 1959. Experiments in hybridizing several species of trout Progressive Fish Culturist 21 (4).

Stenton (J. E.). - 1950. Artificial hybridization of eastern brook trout and lake trout. Canadian Fish culturist 6-20-22.

Vivier (Paul). - 1954. La Pisciculture. Presse Liniversitaire, France. 